\title{
Verbal transformation as a function of boredom susceptibility, attention maintenance, and exposure time
}

\author{
RICHARD S. CALEF, RUTH A. CALEF, EDWARD PIPER \\ DEBRA J. SHIPLEY, and CYNTHIA D. THOMAS \\ West Virginia Wesleyan College, Buckhannon, West Virginia 26201 \\ and \\ E. SCOTT GELLER \\ Virginia Polytechnic Institute and State University, Blacksburg, Virginia 24061
}

\begin{abstract}
Sixteen high-boredom-susceptible (HBS) and 16 low-boredom-susceptible (LBS) college students recorded word distortions on a structured data sheet (i.e., numbered) or on a nonstructured data sheet, resulting in groups of 8 college students who were presented with six neutral words for 6 min each. In the structured situation, the LBS subjects produced significantly more verbal transformations (i.e., word distortions) than HBS subjects, but in the nonstructured situation, there was a trend for HBS subjects to produce a higher verbal transformation rate than that shown by LBS subjects. In addition, all subjects produced significantly more verbal transformations in the last $3 \mathrm{~min}$ as compared to the first $3 \mathrm{~min}$ of exposure time. Attention and arousal notions were considered to interpret these findings.
\end{abstract}

Previous researchers (Evans \& Kitson, 1967; Warren, 1968) have studied subjects' reports of word distortions following the continuous repetition of the same word. Results have shown that a word may undergo illusory changes (verbal transformations) as a function of being repeated aloud a number of times. These transformations have ranged from perceptions of words that rhyme with the actual stimulus to more extreme phonetic distortions such as synonyms or antonyms (Calef, Calef, Kesecker, \& Burwell, 1974).

Theoretically, these word distortions occur because of an absence of psychological and/or physiological "arousal" accompanying auditory repetition. Indeed, Calef et al. (1974), Evans and Kitson (1967), and Warren (1968) obtained results supportive of an "arousal" theory. More specifically, Warren (1968) found verbal transformation (VT) frequency to decrease with phonetic complexity; Evans and Kitson (1967) observed more familiar words to elicit more VTs than less familiar words; and Calef et al. (1974) found more VTs from neutral than taboo words. Thus, it seems that words that elicit more "arousal" maintain the subject's attention longer and yield relatively fewer transformations (e.g., words with less familiarity, more phonetic complexity, and less social acceptability).

Calef, Calef, Piper, Wilson, and Geller (1977a) found that more VTs occurred for young adults than for

Reprint requests should be sent to E. Scott Geller, Department of Psychology, Virginia Polytechnic Institute and State University, Blacksburg, Virginia 24061. children, and that for young adults, VT frequency was directly related to verbal intelligence. These data also seemed to support the "arousal" notion, since repetition should have produced more boredom for the younger and more intelligent adults, resulting in increased VT rate. On the other hand, Calef, Calef, Piper, Wilson, and Geller (1977b) obtained evidence that did not support "arousal" interpretations. Contrary to the "arousal" hypothesis that a neutral stimulus should elicit less "arousal" and more VTs for highboredom-susceptible (HBS) subjects than for lowboredom-susceptible (LBS) subjects, LBS students actually produced significantly more VTs than did HBS students.

Calef et al. (1977a, 1977b) interpreted their inconsistent findings by considering that HBS subjects have a shorter attention span than LBS subjects. Given this assumption, the LBS subjects attended more readily to the repetitive stimuli, thus enabling them to experience more VTs. In other words, the HBS subjects may have had difficulty focusing their attention on the repeating stimuli, thereby precluding them from experiencing word distortions.

It may be critical that Calef et al. (1977b) used a "structured" data sheet. In other words, the data sheets were numbered so that if the subjects did experience a word distortion, they were to place a check mark next to the appropriately numbered trial. This situation could have facilitated attention holding, especially for those subjects whose attention spans are relatively long (i.e., LBS individuals). A major 
purpose of the present study was to investigate whether attention and therefore VT frequency could be manipulated by varying the structure of the data sheet. It was hypothesized that a structured data sheet would produce more VTs for LBS as compared to HBS subjects, because a more structured data sheet should cause the more attentive LBS subjects to focus on the repeating stimuli. However, it is postulated that an unstructured data sheet (one without numbered trials) will not result in the same kind of differences in VT frequency between HBS and LBS subjects, because an unstructured situation should not differentially hold the attention of HBS or LBS subjects. In a neutral situation, the personality variable (boredom susceptibility) should take control and, according to an arousal notion, HBS subjects should produce more VTs than LBS subjects.

A second purpose of the study was to investigate the effects that exposure time of each word would have on VT frequency. Such effects were studied by comparing the number of VTs that occurred during the first $3 \mathrm{~min}$ with those occurring during the last $3 \mathrm{~min}$ of each 6-min word presentation trial. An arousal hypothesis would predict that both HBS and LBS subjects should show more VTs during the last $3 \mathrm{~min}$, since arousal should be minimal at that time.

\section{METHOD}

Seventeen male and 25 female general psychology students served as subjects. For participating in the experiment, subjects received extra points toward their grades in introductory psychology.

The boredom-susceptibility subscale of the sensation-seeking scale (Zuckerman, Kolin, Price, \& Zoob, 1964) was given to 200 introductory psychology students at West Virginia Wesleyan College. The 16 students scoring in the highest and lowest $16 \%$ of the sample were chosen as subjects. The uppermost $16 \%$ of the students were considered HBS and scored above 11 out of a possible 20 points on the boredom scale. The lowest $16 \%$ of the students were defined as LBS and scored below 5 on the scale.

Subjects were divided into LBS and HBS groups and then randomly subdivided into structured data sheet (S) and nonstructured data sheet (NS) groups. Thus, there were four groups of eight subjects each: LBS-S, LBS-NS, HBS-S, and HBS-NS. All subjects were tested at the same time in a large, well lighted classroom. Each word was first recorded on a cassette tape recorder and then rerecorded on a high-fidelity tape recorder, so that the word was repeated at a constant rate of about one word per $3 \mathrm{sec}$ over a 6 -min period. Following the first 3 min of word repetition, a $10-\mathrm{sec}$ rest interval occurred, and then the final $3 \mathrm{~min}$ occurred. The six neutral words were presented to all subjects in the following order: bedroom, telephone, door, people, subjects, and television.

Subjects using the structured data sheet were instructed as follows: "When I switch on the tape recorder, you will hear a word repeated for $6 \mathrm{~min}$ with a 10-sec interlude in the middle. I want you to listen very carefully and quietly, and if you think the word changes, record a check mark after the appropriate numbered trial on your data sheet, even if the change you hear does not resemble a recognizable word. If the word does not change, leave the appropriate numbered trial blank. When you hear the interlude, place a line after the last trial, and return to the first numbered trial. For the final $3 \mathrm{~min}$, if you think the word changes, circle the appropriate numbered trial, and if you do not hear a change in the word, do not circle the appropriate numbered trial."

Subjects using the nonstructured data sheet were given these instructions: "When I switch on the tape recorder, you will hear a word repeated for $6 \mathrm{~min}$ with a 10 -sec interlude in the middle. I want you to listen very carefully and quietly, and if you think the word changes, record a check mark on your paper in a column, even if the change you hear does not resemble a recognizable word. If the word does not change, do not record a check mark. When you hear the interlude, place a line after your last check mark. For the final $3 \mathrm{~min}$, continue to record check marks as you did in the first $3 \mathrm{~min}$, continuing the column on the page." Thus the difference between the S and NS data sheets was that the latter did not have a system for systematically assigning a number to each trial.

After the task instructions, the first word was presented for $6 \mathrm{~min}$ with a 10-sec interval after $3 \mathrm{~min}$. Each word was repeated approximately 60 times per 3 -min segment, with a $1-\mathrm{sec}$ interval between repetitions. The subjects were then given a 3-min rest period between stimuli. This procedure continued until the list of six words was completed.

\section{RESULTS}

Table 1 illustrates average VT frequency per group as a function of boredom susceptibility, attentiveness, (S vs. NS data sheets), and exposure time. As can be seen in Table 1, with $S$ data sheets, LBS subjects showed higher VT rates than HBS subjects; however, only small differences in VTs occurred for both groups under the NS condition. The HBS subjects showed only minor disparities in VT rate under $S$ vs. NS conditions. However, LBS subjects showed more VTs with the numbered than with the nonnumbered data sheet. All groups showed higher VT rates during the last $3 \mathrm{~min}$ than the first $3 \mathrm{~min}$ of exposure time.

A 2 (boredom susceptibility) by 2 (attention maintenance) by 2 (exposure time) analysis of variance was performed on mean VT frequencies. The analysis yielded a significant main effect of exposure time $[F(1,28)=34.82, p<.01]$ and a significant Boredom Susceptibility by Attentiveness interaction $[F(1,28)=$ $5.25, \mathrm{p}<.05]$. No other effects were reliable ( $\mathrm{ps}>.05)$. A number of paired comparisons using the Tukey a test were performed on the data in order to interpret the significant interaction. The test showed a significantly

Table 1

Group Average Verbal Transformations as a Function of Boredom Susceptibility, Attention Maintenance, and Exposure Time

\begin{tabular}{|c|c|c|c|c|}
\hline \multirow[b]{3}{*}{$\begin{array}{c}\text { Boredom } \\
\text { Susceptibility }\end{array}$} & \multicolumn{4}{|c|}{ Attention Maintenance } \\
\hline & \multicolumn{2}{|c|}{ Structured Data Sheet } & \multicolumn{2}{|c|}{ Nonstructured Data Sheet } \\
\hline & $\begin{array}{l}\text { First } \\
3 \mathrm{~min}\end{array}$ & $\begin{array}{c}\text { Last } \\
3 \mathrm{~min}\end{array}$ & $\begin{array}{c}\text { First } \\
3 \text { min }\end{array}$ & $\begin{array}{l}\text { Last } \\
3 \mathrm{~min}\end{array}$ \\
\hline High & 19.22 & 41.67 & 22.86 & 38.60 \\
\hline Low & 28.42 & 55.02 & 18.42 & 32.17 \\
\hline
\end{tabular}


$(p<.05)$ higher VT rate for LBS than HBS subjects under the $\mathrm{S}$ data sheet condition; however, no differences due to boredom susceptibility occurred under the NS condition ( $p>.05)$. Between-groups Tukey a tests showed no differences in VT rate as a function of the attention condition for the HBS subjects, but LBS subjects showed significantly more VTs with the $S$ data sheet than with the NS data sheet $(p<.01)$.

\section{DISCUSSION}

The results of the present study replicate the Calef et al. (1977b) study in that LBS subjects showed more VTs than HBS subjects in an attention-maintaining situation. More importantly, however, the present results indicated that the variable that was shown to facilitate VT rate for LBS subjects was presumed to influence attention, since in a nonattentionmaintaining situation, Group LBS did not produce a greater VT rate than that shown by Group HBS. The observation that LBS subjects showed higher VT rates in an attentionmaintaining situation as compared to one that was not conducive to maintaining attention, but that HBS subjects produced approximately the same VT rate regardless of the attentionmaintaining situation lends even more support to the notion that attention was the relevant variable in the Calef et al. (1977b) study.

The present data are unclear as to whether boredom susceptibility is related to arousability. That is, in the nonattention-maintaining situation, there were no significant differences in the average number of VTs between HBS and LBS subjects. According to an arousal notion, the HBS subjects should have shown a higher VT rate than the LBS subjects, because the former group should have been less aroused, which in turn should have produced more auditory distortions. However, there was a trend for HBS subjects to show more VTs than the number shown by LBS subjects.
The results that all groups reported more VTs during the last $3 \mathrm{~min}$ than during the first $3 \mathrm{~min}$ support an arousal hypothesis. The arousal notion would predict more VTs for all subjects during a time period of the least arousal (the last $3 \mathrm{~min}$ ).

Further research should investigate whether other attentionmaintaining variables affect difference in VT rate. For example, one may expect LBS subjects to produce the most VTs in a situation where every subject is tested individually with a numbered data sheet, since this situation should increase attention and bring out the attentive attributes of LBS subjects.

\section{REFERENCES}

Calef, R. S., Calef, R. A., Kesecker, M. P., \& Burwell, R. Verbal transformations of "stabilized" taboo and neutral words. Perceptual and Motor Skills, 1974, 38, 177-178.

Calef, R. S., Calef, R. A., Piper, E. H., Wilson, S. A., \& GELLER, E. S. Imagined verbal transformations as a function of age and verbal intelligence. Bulletin of the Psychonomic Society, 1977, 10, 109-110. (a)

Calef, R. S., Calef, R. A., Piper, E. H., Wilson, S. A., \& GelleR, E. S. Verbal transformations and boredom susceptibility. Bulletin of the Psychonomic Society, 1977, 10, 367-368. (b)

Evans, C. R., \& Kitson, A. An experimental investigation of the relation between the "familiarity" of a word and the number of changes in its perception which occur with repeated presentation as a "stabilized" auditory image. National Physical Laboratory Auto Report, 1967, Whole No. 36.

W ARREN, R. M. Verbal transformation effect on auditory perceptual mechanisms. Psychological Bulletin, 1968, 70, 261-270.

Zuckerman, M., Kolin, E. A., Price, L., \& Zoob, I. Development of a sensation-seeking scale. Journal of Consulting Psychology, 1964, 28, 477-482.

(Received for publication November 11, 1978.) 